\title{
CHAOS: THE EVOLUTION OF A DEFINITION
}

\author{
Pat Touhey
}

\section{Introduction}

Chaos, a buzzword for our times. But what is it? To a scientist, chaos is a natural phenomenon. It has been around forever but folklore has it that the idea was first articulated by Edward Lorenz [4] in his investigations of the weather. ${ }^{1}$ Basically it is the seemingly paradoxical result that a well understood deterministic process may be practicably unpredictable. That is to say, given a perfectly wonderful mathematical model of some physical phenomenon, the perfectly wonderful model may prove to be absolutely useless in that we can not utilise it to predict the future states of the physical process which we have so successfully modelled. The best known and most popular example of this paradoxical result is the so-called "Butterfly Effect". Imagine that we have in hand a deterministic model for the weather; that is, if we plug in the appropriate variables, e.g. time, position, wind velocity, barometric pressure, air temperature, etc, out pops a correct meteorological forecast. Nevertheless our model may prove useless. A small error in input, such as neglecting the effect that the flapping of a butterfly's wings has upon wind speed, may in fact result in catastrophic errors in the future output. And in practice the accuracy of our measurements is always limited by the precision of our instruments. Thus our input is always subject to small errors. Hence, chaos. We leave the metaphysical implications of this example to the philosophically inclined reader and return to the realm of mathematics.

\footnotetext{
1 In a recent book review, [3], Freeman Dyson attributes the discovery of "chaos" to Mary Cartwright and J. E. Littlewood in 1945.
} 


\section{Devaney's definition of chaos}

A mathematical definition needs no justification. Yet, to be of interest, it should have some relation to an interesting problem. Thus a definition of chaos should in some sense embody the essence of the scientific problem encountered in investigations of the weather as described above. It follows that a key ingredient of any definition of chaos should be unpredictability, by which we mean that the predicted output of a chaotic system should be seriously affected by even the slightest change in the inputted data. Phrased succinctly, it should possess sensitive dependence on initial conditions. Additionally we wish to insure that our chaotic system cannot be broken down and decomposed into subsytems that do not interact. This is consistent with our meteorological example; a monsoon in Calcutta today will eventually have an effect on the weather in Galway. Thus our definition should also include a condition of indecomposability. Lastly in the midst of this complicated behaviour there should be a semblance of order, an element of regularity, in much the way that the seasons cycle and the air temperature rises and falls daily. These three conditions, sensitivity to initial conditions, indecomposability and an element of regularity, motivate the definition of chaos as given by Devaney in [2].

But before we state that definition we need to introduce some terminology and notation. Throughout we assume that $X \equiv(X, d)$ is a metric space and that $f: X \rightarrow X$ is a continuous map ${ }^{2}$ with $f^{n}: X \rightarrow X$ being its $n$-th iterate.

Definition 2.1 Given $f: X \rightarrow X$, the point $x$ is a periodic point of $f$ with primitive period $n$, if $f^{n}(x)=x$ but $f^{k}(x) \neq x$ for all $k, 1 \leq k \leq n-1$.

In order to provide that a mapping $f: X \rightarrow X$ has an element of regularity we will require that the set of all periodic points of $f$ be dense in $X$.

Next we define the concept of topological transitivity which will be used to ensure that a map is indecomposable.

\footnotetext{
2 We should emphasize that we require chaotic maps to be continuous, whereas Devaney only requires that they be functions.
} 
Definition 2.2 Given $f: X \rightarrow X$, we say that $f$ is transitive if for all non-empty open subsets $U$ and $V$ of $X$ there exists a non-negative integer $k$ such that $f^{k}(U) \cap V \neq \emptyset$.

It is easy to see that transitivity guarantees the existence of a point in $U$ which will, after some number of iterates of the map $f$, reach the set $V$. And now we turn to the definition of sensitive dependence on initial conditions.

Definition 2.3 Given $f: X \rightarrow X$ we say that $f$ has sensitive dependence on initial conditions if there exists a $\delta>0$ such that, for any $x \in X$ and any $\epsilon>0$, there exists a $y \in X$ and a nonnegative integer $k$, such that $d(x, y)<\epsilon$ but $d\left(f^{k}(x), f^{k}(y)\right)>\delta$.

The $\delta$ in the definition above is referred to as the sensitivity constant. Devaney's definition of chaos can now be given.

Definition 2.4 We say that $f: X \rightarrow X$ is chaotic on $X$ if

- $f$ is transitive

- the set of periodic points of $f$ is dense in $X$

- $f$ has sensitive dependence on initial conditions

We emphasize that this is only one of many possible mathematical definitions of chaos, all of which attempt to capture the essence of the scientific phenomenon called chaos. But Devaney's definition has certainly become one of the most popular. It is also the most purely topological and thus in some sense the simplest definition. Where other definitions rely upon notions like positive Lyapunov exponents or Kolmogorov-Sinai metric entropy, Devaney's definition utilises concepts familiar to any student of basic point set topology. However this definition may be made even simpler. Interestingly it turns out that the first two conditions, that $f$ be transitive and that the set of periodic points of $f$ be dense in $X$, are enough to imply that $f$ has sensitive dependence on initial conditions. That surprising fact, due to Banks, Brooks, Cairns, Davis and Stacey, is the subject of our next section.

\section{The La Trobe definition of chaos}

A departmental seminar on Chaotic Dynamical Systems at La Trobe University in Melbourne, Australia resulted in the publication of an elegant result simplifying the definition of chaos [1]. 
Theorem 3.1 If $f: X \rightarrow X$ is transitive and the set of periodic points of $f$ is dense in $X$, then $f$ has sensitive dependence on initial conditions.

Proof: The proof we give here is only slightly different than the one given in [1]. Start by assuming that the space $X$ has a pair of disjoint orbits. If this were not the case our metric space would be quite uninteresting. In fact we leave it to the reader to show that the space would consist of a single periodic orbit.

Assuming we have a pair of disjoint orbits we note that there is a positive distance, $\delta_{0}$, between those two orbits. This follows easily from the fact that both orbits consist of only a finite number of points. Our sensitivity constant, $\delta$, will depend on $\delta_{0}$. In fact $\delta \equiv \frac{\delta_{0}}{8}$.

Now consider any point $x \in X$. We need to show that for any $\epsilon>0$, there exists a point $z$ and a non-negative integer $k$, such that $z \in N_{\epsilon}(x)$ but $d\left(f^{k}(x), f^{k}(z)\right)>\delta$, where $N_{\epsilon}(x)$ stands for the $\epsilon$-neighbourhood of $x$.

Towards that end, recall that there exists a pair of periodic orbits separated by a distance of at least $8 \delta$. Obviously the point $x$ can not be close to both of them. Let $q$ be a point in the periodic orbit, $O_{f}^{+}(q)$, which is at least $4 \delta$ from $x$. We will assume that the orbit has period $j$, hence

$$
O_{f}^{+}(q) \equiv\left\{q, f(q), f^{2}(q), \ldots, f^{j-1}(q)\right\}
$$

Now since $X$ is a metric space, in fact Hausdorff would suffice, we may separate any finite set of points by disjoint open sets. In particular we may separate the set $\{x\} \cup O_{f}^{+}(q)$ by disjoint open sets, $V_{x}, V_{q}, V_{f(q)}, \ldots, V_{f^{j-1}(q)}$. Now let $V_{x}$ be intersected with $N_{\epsilon}(x)$ to form an open set $V$. Then to insure that all of these sets are small enough we will intersect each with an appropriate $\delta$-neighbourhood to arrive at the following collection of disjoint 
open sets,

$$
\begin{aligned}
N= & V \cap N_{\delta}(x) \\
U_{0}= & V_{q} \cap N_{\delta}(q) \\
U_{1}= & V_{f(q)} \cap N_{\delta}(f(q)) \\
& \cdots \\
U_{j-1}= & V_{f^{j-1}(q)} \cap N_{\delta}\left(f^{j-1}(q)\right) .
\end{aligned}
$$

We will show that $N$ contains a point that eventually, after a finite number of iterates, separates from $x$ by a distance greater than $\delta$. But first note that the open set $N \backslash\{x\}$ must contain a periodic point $p$. This follows from the density of periodic points. The periodic point $p$ has some finite period $m$. Thus $p$ will return to the set $N$ every $m$ iterates.

Now let us return to our periodic orbit $O_{f}^{+}(q)$. We want to create a sequence of open sets with a particularly nice property. Begin by letting $W_{0} \equiv U_{0}$, the open set containing $q$. Since $q$ has period $j$ it's clear that the point $f^{-1}(q) \in U_{j-1}$. Let $W_{1} \equiv$ $f^{-1}\left(W_{0}\right) \cap U_{j-1}$. Note that $W_{1}$ is open and non-empty. This follows from the fact that $f^{-1}\left(W_{0}\right)$ and $U_{j-1}$ are both open sets containing $f^{-1}(q)$. And $W_{1}$ has the particularly nice property that its image is contained in $W_{0}$, i.e. $f\left(W_{1}\right) \subset W_{0}$. This is of course nothing more than the freshman calculus characterization of continuity. Given a point contained in an open set, $f^{-1}(q) \in U_{j-1}$, and a target containing the point's image, $q \in W_{0}$, there exists an open neighbourhood about the point, $W_{1} \subset U_{j-1}$, such that $f\left(W_{1}\right) \subset W_{0}$. Repeating this process we create a sequence of open sets, $\left\{W_{i}\right\}_{i=0, \ldots, m}$, where

$$
\begin{aligned}
W_{0} \equiv & U_{0} \\
W_{1} \equiv & f^{-1}\left(W_{0}\right) \cap U_{j-1} \\
& \cdots \\
W_{i} \equiv & f^{-1}\left(W_{i-1}\right) \cap U_{j-i} \\
& \cdots \\
W_{m} \equiv & f^{-1}\left(W_{m-1}\right) \cap U_{j-m} .
\end{aligned}
$$


The key point is that the entire open set $W_{m}$ will shadow the periodic orbit $O_{f}^{+}(q)$ for at least $m$ iterates. ${ }^{3}$ More precisely,

$$
\begin{aligned}
& f\left(W_{m}\right) \subset W_{m-1} \\
& f^{2}\left(W_{m}\right) \subset f\left(W_{m-1}\right) \subset W_{m-2} \\
& \ldots \\
& f^{m}\left(W_{m}\right) \subset f^{m-1}\left(W_{m-1}\right) \subset \ldots \subset f\left(W_{1}\right) \subset W_{0} .
\end{aligned}
$$

Now by transitivity there must be a point $y$ in $N$, different from $x$ or $p$, which upon iteration will eventually, say after $k$ iterates, wind up in the open set $W_{m}$. And once that point reaches $W_{m}$ we know that it will shadow the periodic orbit $O_{f}^{+}(q)$ for at least the next $m$ iterates. But while $y$ is shadowing $O_{f}^{+}(q)$ the periodic point $p$ must return back to $N$. Thus at some iterate $i$, where $k \leq i \leq k+m, f^{i}(y)$ and $f^{i}(p)$ are far apart. In fact since $N$ and the periodic orbit $O_{f}^{+}(q)$ are at least $3 \delta$ apart we can state that $d\left(f^{i}(y), f^{i}(p)\right)>2 \delta$.

But where is $x$ ? Or more precisely, where is $f^{i}(x)$ ? Clearly it can't be close to both of $f^{i}(y)$ and $f^{i}(p)$. Thus we have found a point contained in $N$, be it $p$ or $y$, with the property that after $i$ iterates of $f$ that point and $x$ are separated by a distance greater than $\delta$.

The marvellous fact about this last theorem is that a pair of topological conditions, transitivity and density of periodic points, have been shown to have decidedly metric consequences. This mathematically elegant result has completely removed the concept of sensitive dependence on initial conditions from the definition of chaos. Paradoxically, the hallmark of chaos is superfluous in its definition.

3 To be completely honest we should note that in the course of the proof we have tacitly assumed that the length of the periodic orbit $O_{f}^{+}(q)$ is greater than the period of the point $p$. There is no great harm in so doing and it helps to insure that the simplicity of the argument is not lost in a "debauche of indices". 


\section{The C. U. N. Y. definition of chaos}

Definition 4.1 A mapping $f: X \rightarrow X$ is chaotic if every pair of non-empty open subsets of $X$ shares a periodic forward orbit. That is, given $U$ and $V$, non-empty open subsets of $X$, there exists a periodic point $p \in U$ and a non-negative integer $k$, such that $f^{k}(p) \in V$.

This would appear to be a simpler definition of chaos but is it equivalent to the La Trobe version? That question is resolved by our next proposition.

Proposition 4.2 $f: X \rightarrow X$ is chaotic if and only if $f$ is transitive and the periodic points of $f$ are dense in $X$.

Proof: If $f$ is chaotic on $X$ then every pair of non-empty open sets shares a periodic orbit. In particular, every non-empty open set must contain a periodic point and so the periodic points of $f$ are dense in $X$. The transitivity of $f$ follows from the definition of chaos since every pair of non-empty open sets share a forward orbit.

Now let us assume that $f$ is transitive and has a dense set of periodic points. Given any pair of non-empty open sets $U, V \subset X$ there exists $u \in U$ and a non-negative integer $k$ such that $f^{k}(u) \in$ $V$. This is of course by transitivity. Now define $W \equiv f^{-k}(V) \cap U$. Note that $W$ is open and non-empty since it is the intersection of two open sets and $u$ is an element of both of them. It is also clear that $W$ has the property that $f^{k}(W) \subset V$. But the periodic points of $f$ are assumed to be dense in $X$, hence $W$, being non-empty and open, must contain a periodic point $p$. Thus we have shown that there exists a periodic point $p \in W \subset U$ with the property that $f^{k}(p) \in f^{k}(W) \subset V$. This of course implies that $f$ is chaotic.

This new definition of chaos arose several years ago as a result of a collaboration between the author and John Taylor while we were colleagues at Lehman College of The City University of New York. With it in hand it is possible to state a variety of equivalent formulations of the concept of chaos, thus restoring some of the intuition that was lost with the removal of sensitive dependence from the stage. These results first appeared in [5].

It can now be shown that any finite number of non-empty 
open sets will also share a periodic orbit whenever $f$ is chaotic.

Proposition 4.3 Let $X$ be a metric space and $f: X \rightarrow X$ a chaotic mapping. Then any finite collection of non-empty open subsets of $X$ shares a periodic forward orbit.

Proof: Let $N$ be the number of non-empty open subsets in our finite collection. If $N=2$, the result follows from the definition of a chaotic mapping. We will proceed by induction on $N$. Thus assume that the result holds for $N=n$. We will show that it holds for $n+1$ non-empty open subsets.

First note that without loss of generality we may assume that the collection consists of $n+1$ disjoint subsets. If not, then at least a pair of the non-empty open subsets intersect in an open subset. Replace the pair by their intersection to form a collection of $n$ non-empty open subsets which by our induction hypothesis must share a periodic forward orbit. Clearly this orbit is shared by the original collection of $n+1$ subsets.

So label the disjoint collection of $n+1$ subsets as $U, U_{0}, U_{1}, \ldots$, $U_{n-1}$. It follows from our induction hypotheses that the $n$ subsets, $U_{0}, U_{1}, \ldots U_{n-1}$, must share a periodic orbit, i.e. there exists a periodic point $p \in U_{0}$, such that $O_{f}^{+}(p) \cap U_{i} \neq \emptyset, 0 \leq i \leq n-1$. Upon iteration the point $p$ will first intersect one of the $U_{i}$ 's for some value, $k_{1}$, of the iterate. Thus, by relabelling the $U_{i}$ 's if necessary, we have $f^{k_{1}}(p) \in U_{1}$. Continuing in this fashion we will arrive at the next iterate, $f^{k_{2}}(p), 0<k_{1}<k_{2}$, intersecting one of the remaining $n-2$ open subsets. This subset is designated $U_{2}$.

Eventually we will have

$$
f^{k_{i}}(p) \in U_{i} \quad \text { for all } i=0,1, \ldots, n-1
$$

where $0=k_{0}<k_{1}<\cdots<k_{n-1}$. Now we will define another collection of non-empty open subsets with a particularly nice property. Let $W_{0} \equiv U_{n-1}$. Now consider

$$
W_{1} \equiv f^{-\left[k_{n-1}-k_{n-2}\right]}\left(W_{0}\right) \cap U_{n-2}
$$

$W_{1}$ is a non-empty open subset contained in $U_{n-2}$, clearly open because it is the intersection of two open subsets and obviously in 
$U_{n-2}$. That it is non-empty follows from the facts that

$$
\begin{aligned}
& \quad f^{k_{n-2}}(p) \in U_{n-2} \\
& \text { and } \\
& \qquad f^{k_{n-2}}(p)=f^{-\left[k_{n-1}-k_{n-2}\right]}\left(f^{k_{n-1}}(p)\right) \in f^{-\left[k_{n-1}-k_{n-2}\right]}\left(W_{0}\right) \\
& \text { since } \\
& \qquad f^{k_{n-1}}(p) \in W_{0} .
\end{aligned}
$$

Hence $f^{k_{n-2}}(p) \in W_{1}$. Also note that $W_{1}$ has the particularly nice property that $f^{\left[k_{n-1}-k_{n-2}\right]}\left(W_{1}\right) \subset W_{0}$. Again this is nothing more than the freshman calculus characterization of continuity with regard to $f^{\left[k_{n-1}-k_{n-2}\right]}$.

Now continuing in this fashion we define

$$
W_{i} \equiv f^{-\left[k_{n-i}-k_{n-(i+1)}\right]}\left(W_{i-1}\right) \cap U_{n-(i+1)}
$$

for $i=1,2, \ldots, n-1$. As above, each $W_{i}$ will be non-empty, open and contained in $U_{n-(i+1)}$. In addition we have the particularly nice property that

$$
f^{\left[k_{n-i}-k_{n-(i+1)}\right]}\left(W_{i}\right) \subset W_{i-1}
$$

for $i=1,2, \ldots, n-1$. Now it is easy to find a periodic orbit which wends itself through our original collection of $n+1$ nonempty open subsets, $\left\{U, U_{0}, U_{1}, \ldots, U_{n-1}\right\}$. The procedure rests on the fact that the entire open set $W_{n-1}$ will shadow the periodic orbit $O_{f}^{+}(p)$ for at least $k_{n-1}$ iterates. But since $U$ and $W_{n-1}$ are both open, they must share a periodic forward orbit. Thus there exists a periodic point $p^{\prime} \in U$ and a positive integer $q$ such that $f^{q}\left(p^{\prime}\right) \in W_{n-1} \subset U_{0}$. But then, by our particularly nice property, 
the subsequent iterates of $p^{\prime}$ must pass through all of the $U_{i}$ 's.

$$
\begin{aligned}
& f^{q}\left(p^{\prime}\right)=f^{\left[q+k_{0}\right]}\left(p^{\prime}\right) \in W_{n-1} \subset U_{0} \\
& f^{q+k_{1}}\left(p^{\prime}\right)=f^{\left[k_{1}-k_{0}\right]}\left(f^{\left[q+k_{0}\right]}\left(p^{\prime}\right)\right) \subset f^{\left[k_{1}-k_{0}\right]}\left(W_{n-1}\right) \\
& \subset\left(W_{n-2}\right) \subset U_{1} \\
& f^{q+k_{i}}\left(p^{\prime}\right)=f^{\left[k_{i}-k_{i-1}\right]}\left(f^{\left[q+k_{i-1}\right]}\left(p^{\prime}\right)\right) \subset f^{\left[k_{i}-k_{i-1}\right]}\left(W_{n-i}\right) \\
& \subset W_{n-(i+1)} \subset U_{i} \\
& f^{q+k_{n-1}}\left(p^{\prime}\right)=f^{\left[k_{n-1}-k_{n-2}\right]}\left(f^{\left[q+k_{n-2}\right]}\left(p^{\prime}\right)\right) \subset f^{\left[k_{n-1}-k_{n-2}\right]}\left(W_{1}\right) \\
& \subset W_{0}=U_{n-1} \text {. }
\end{aligned}
$$

Hence the forward orbit of $p^{\prime}, O_{f}^{+}\left(p^{\prime}\right)$, intersects each of $U, U_{0}$, $U_{1}, \ldots, U_{n-1}$.

However we can say even more. Not only will a chaotic map link any finite number of open sets together, it will link them via an infinite number of periodic orbits.

Corollary 4.4 Let $X$ be a metric space and $f: X \rightarrow X$ a chaotic mapping. Then any finite collection of non-empty open subsets of $X$ share infinitely many periodic forward orbits.

Proof: Assume the existence of a finite collection $\left\{U_{i}\right\}_{i=1, \ldots, n}$ of non-empty open subsets sharing only a finite number of periodic forward orbits. Define $P$ to be the set consisting of the union of the points in these shared periodic forward orbits. Since each periodic forward orbit contains a finite number of points the union of finitely many such orbits must be finite. Hence $P$ is a finite set. We now define another collection of non-empty open subsets $\left\{W_{i}\right\}_{i=1, \ldots, n}$ by $W_{i} \equiv U_{i} \backslash P$. It's clear that each $W_{i} \subset U_{i}$, and each $W_{i}$ is non-empty and open since removing the finite set of points, $P$, from the open set $U_{i}$ leaves us with a non-empty open set. Thus by Proposition 4.3 there must be a periodic forward orbit shared by the collection $\left\{W_{i}\right\}_{i=1, \ldots, n}$. This new orbit is clearly not contained 
in $P$. On the other hand this orbit obviously passes through the original collection $\left\{U_{i}\right\}_{i=1, \ldots, n}$ of non-empty open subsets since each $W_{i} \subset U_{i}$. This contradiction proves our result.

Proposition 4.5 Let $X$ be a metric space and $f: X \rightarrow X$ a mapping. The following are equivalent:

i) $f$ is chaotic.

ii) $f$ is topologically transitive and has a dense set of periodic points.

iii) any finite collection of non-empty open sets of $X$ share a periodic orbit.

iv) any finite collection of non-empty open sets of $X$ share infinitely many periodic orbits.

Proof: That i) $\Longleftrightarrow$ ii) $\Longrightarrow$ iii) $\Longrightarrow$ iv) has been shown above. But the fact that any finite collection of non-empty open sets contained in $X$ share infinitely many periodic orbits clearly implies that any pair of open sets share a periodic orbit. Thus iv) $\Longrightarrow$ i).

\section{References}

[1] J. Banks, J. Brooks, G. Cairns, G. Davis and P. Stacey, On Devaney's definition of chaos, Amer. Math. Monthly 99 (1992), 332-334.

[2] Robert L. Devaney, An Introduction to Chaotical Dynamical Systems. Addison-Wesley: 1989.

[3] Freeman Dyson, Review of Nature's Numbers, Amer. Math. Monthly 103 (1996), 610-12.

[4] Edward Lorenz, Deterministic non-periodic flow, J. Atmos. Sci.20 (1963), 130-41.

[5] Pat Touhey, Yet another definition of chaos, Amer. Math. Monthly 104 (1997), 411-414.

Pat Touhey

Department of Mathematics and Computer Science,

College Misericordia,

Dallas, Pennsylvania 18612,

USA. 